\title{
CORRESPONDENCE:
}

\section{Distinguishing variability from uncertainty}

To the Editor - Uncertainty in climate predictions, and especially in tippingpoints towards dangerous climate change, is not only a challenge for science communication ${ }^{1}$ but also for triggering collective action as outlined by Barrett and Dannenberg ${ }^{2}$.

Uncertainty is a measure of unexplained variation, and can be partly caused by measurement errors, and partly by our lack of understanding about cause and effect. But predictions of climate change, and approaches to its mitigation, do not only carry uncertainties in the magnitude of responses, they also entail significant natural variability in time and space ${ }^{3.4}$. Importantly, this spatial and temporal variability will not shrink with scientific progress. Embracing the difference and clearly distinguishing between these two sources of variation is therefore critically important for science communication as well as for collective and policy action (see Fig. 1).

The following example illustrates the importance of this distinction. When experts were asked about the scientific certainty regarding the extent to which different land cover and management options affect greenhouse gas emissions, the certainty of the answers ranged between medium and low ${ }^{5}$. Even with 246 comparisons drawn from the scientific literature about the effects of switching to reduced tillage, the scientific certainty was judged to be only 'medium'. When averaged over the entire United States, the increase in soil organic carbon was calculated as $1.22 \mathrm{tCO}_{2}$ equivalent ha ${ }^{-1}$ but ranged between a decrease of $0.24 \mathrm{tCO}_{2}$ equivalent $\mathrm{ha}^{-1}$ and an increase of $3.22 \mathrm{tCO}_{2}$ equivalent ha-1 (ref. 6). What at first glance may seem to be uncertainty, reveals itself as spatial heterogeneity: soil carbon contents respond dramatically differently from place to place. In the cool, moist northern and northeastern US, reduction of soil tillage is expected to cause carbon losses, whereas increases in soil carbon might be expected in the Corn Belt ${ }^{4}$.

This is a flaw in science communication that blurs the lines of scientific knowledge: we need to clearly distinguish between a lack of process understanding and the failure to adequately capture the heterogeneity of



Figure 1 | Proposed terminology to distinguish between a reduction in confidence due to unexplained variation, such as uncertainty, and reduction due to already explained and understood variation, such as spatial or temporal variability. Progress in science will decrease uncertainty (though probably not eliminate it), but will not decrease total variation.

responses. The average response in the described cases is nearly meaningless. The large 'error' is not primarily that we do not know 'what' happens, but that we fail to distinguish 'where, 'when' and 'as a result of what'. Even though climate science explicitly describes this variability, communication often fails to capture it, not only with the public but even among scientists. This is to some degree a result of catering to the request for global predictions or that are easily scalable, and is met by showing simple cause-and-effect relationships.

Ecological sciences, by comparison, are specifically targeting responses with high variation. In fact, variability in time and space is the 'raw material' to which explanatory variables are applied, for example through variance partitioning ${ }^{7}$. By disentangling variation in environmental behaviour, the underlying mechanisms can be better understood.

This also applies to climate science, which will benefit from borrowing approaches from other scientific disciplines. Known variation should therefore never be called uncertainty, but explained as variability (Fig. 1). Greater collective action, but also policy changes and effective communication of climate science, will depend on capturing not only unexplained uncertainty but also natural and perfectly explainable variability.

References

1. Katz, W. R. et al. Nature Clim. Change 3, 769-771 (2013).

2. Barrett, S. \& Dannenberg, A. Nature Clim. Change 4, 36-39 (2014).

3. Deser, C., Knutti, R., Solomon, S. \& Phillips, A. S. Nature Clim. Change 2, 775-779 (2012).

4. Ogle, S. M., Swan, A. \& Paustian, K. Agric. Ecosys. Environ. 149, 37-49 (2012)

5. Eagle, A. J. et al. T-AGG Survey of Experts: Scientific Certainty Associated with GHG Mitigation Potential of Agricultural Land Management Practices (Nicholas Institute for Environmental Policy Solutions, 2011).

6. Eagle, A. J. et al. Greenhouse Gas Mitigation Potential of Agricultural Land Management in the United States: A Synthesis of the Literature (Nicholas Institute for Environmental Policy Solutions, 2012).

7. Legendre, P. \& Legendre, L. Numerical Ecology 3rd edn (Elsevier, 2012).

Johannes Lehmann ${ }^{1 \star}$ and Matthias Rillig ${ }^{2,3}$ ${ }^{1}$ Atkinson Center for a Sustainable Future, Department of Crop and Soil Sciences, Cornell University, Ithaca, New York 14853, USA. 2Institute of Biology, Freie Universität, 14195 Berlin, Germany. ${ }^{3}$ Berlin-Brandenburg Institute of Advanced Biodiversity Research (BBIB),

14195 Berlin, Germany.

*e-mail: CL273@cornell.edu 